Depression and Anxiety Vol 32, 6, pp437-444 June 2015

http://dx.doi.org.ezproxy.canterbury.ac.nz/10.1002/da.22341

Accession Number: 2015-07060-001

\title{
Changes in neuropsychological function after treatment with metacognitive therapy or cognitive behaviour therapy for depression
}

Samantha J Groves ${ }^{1,3}$, Richard J Porter ${ }^{1,2}$, Jennifer Jordan ${ }^{1,2}$, Robert Knight ${ }^{3}$, Janet D Carter ${ }^{4}$, Virginia VW McIntosh ${ }^{2}$, Kumari Fernando ${ }^{5}$, Christopher MA Frampton ${ }^{1}$, Roger T Mulder ${ }^{1,2}$, Cameron Lacey $^{1,2}$, and Peter R Joyce ${ }^{1}$

${ }^{1}$ Department of Psychological Medicine, University of Otago, Christchurch, New Zealand

${ }^{2}$ Canterbury District Health Board, New Zealand

${ }^{3}$ Psychology Department, University of Otago, Dunedin

${ }^{4}$ Psychology Department, University of Canterbury, New Zealand

${ }^{5}$ Department of Psychological Medicine, University of Otago, Dunedin, New Zealand

Corresponding author: Dr Richard Porter, Department of Psychological Medicine, University of Otago, Christchurch, P.O. Box 4345, Christchurch 8140, New Zealand

Phone: +643-3720400Ｆax +643-3720407Ｅmail: richard.porter@otago.ac.nz

Short title: Neuropsychological change after cognitive therapies

Keywords: cognitive behaviour therapy, depression, cognition, treatment

\section{Conflict of interest statement}


Dr. Jordan reports grants from New Zealand Lottery Board Health fund (grant 279032), grants from University of Otago Research Fund (grant ORG0109-0310) during the conduct of the study; none of the other authors has any conflict of interest in relation to this manuscript. 


\begin{abstract}
Background: Metacognitive therapy (MCT) is an innovative treatment model addressing patterns of negative thinking seen in emotional disorders. Unlike cognitive behaviour therapy (CBT), MCT has strategies targeting dysfunctional cognitive and metacognitive processes underlying perseverative thinking patterns and attentional biases. The aim of this pilot study was to compare changes in neuropsychological functioning related to executive function and attention in outpatients with depression following treatment with MCT or CBT.
\end{abstract}

Methods: 48 participants referred for outpatient treatment of depression were randomised to 12 weeks of MCT $(n=23)$ or CBT $(n=25)$. Mood severity and neuropsychological functioning were assessed at pre-treatment, 4 weeks and at end treatment (12 weeks).

Results: There were no significant group differences at pre-treatment or 4 weeks on any neuropsychological test, although overall both groups showed a small improvement by 4 weeks. At end treatment, the MCT group demonstrated significantly greater improvement in performance on a task requiring spatial working memory and attention than the CBT group. Changes in executive functioning and attention were independent of change in mood symptoms. Conclusions: MCT may have an advantage over CBT in improving aspects of executive function, including attention. MCT's emphasis on attentional training and flexible control of thinking may have a beneficial effect on neuropsychological functioning, consistent with the purported mechanism of action. 


\section{Introduction}

Major depressive disorder (MDD) is characterised by significant neuropsychological impairment, with deficits being pronounced in executive function processes and attention. Executive function involves the regulation of cognitive activity encompassing capacities such as planning, reasoning, information updating and monitoring, working memory, and mental flexibility including attentional deployment, set shifting, initiating, maintaining or inhibiting responses. ${ }^{12}$ Although the relation between mood and neuropsychological symptoms in MDD is complex, the consensus is that in a substantial proportion of patients, a significant degree of neuropsychological impairment remains even after mood symptoms measured by mood rating scales have improved. ${ }^{3,4}$

Neuropsychological deficit has also been closely linked to functional outcomes in mood disorders ${ }^{5}$ and this may be a factor associated with poorer treatment outcomes ${ }^{6}$ and an increased likelihood of future relapse. ${ }^{7}$ Therefore, integrating specific cognitive remediation strategies to directly target executive function and attention deficits may be particularly important when treating mood disorders.

Cognitive Behaviour Therapy (CBT) has demonstrated efficacy, is recommended in treatment guidelines and often referred to as the 'gold standard' of psychotherapies for depression (e.g. ${ }^{8}$ ). Despite this, only around half of patients respond to treatment and relapse is common ${ }^{9}$. The primary goal of CBT is to challenge the content of negative thoughts believed to be maintaining depressive affect. ${ }^{10} \mathrm{CBT}$ does not specifically target the executive function and attention deficits which are increasingly recognised as playing a role in the aetiology and recurrence of depression (e.g. $\left.{ }^{11}\right)$.

Metacognitive Therapy (MCT) is a relatively new psychotherapy focussing on altering unhelpful patterns of perseverative thinking (rumination and worry), enabling disengagement 
from unhealthy attentional deployment and coping strategies comprising the cognitive attentional syndrome (CAS), theorised to be an important maintaining factor in depression. ${ }^{12}$ The CAS includes rumination and worry thought processes, a pattern of focussing attention on threat, and use of unhelpful coping strategies that inadvertently extend rather than stop negative thinking. These strategies involve cognitive, emotional and behavioural avoidance including substance use or bingeing to avoid uncomfortable emotion. A key constituent of MCT is the Attention Training Technique $\left(\mathrm{ATT}^{13}\right)$ which is based, in part, on knowledge of the executive dysfunction believed to underlie depression. ATT is an auditory attention task involving practice of selective and divided attention and attention switching. Attention control strategies in MCT aim to ameliorate deficits in executive function and attention processes by enhancing attentional capacity and cognitive flexibility - this enables disengagement from mood-congruent stimuli (in conjunction with Wells' detached mindfulness strategy), thereby alleviating depressive symptomology. ${ }^{12}$ Detached mindfulness, one of MCT's other core strategies has been defined by Wells as "a state of awareness of internal events, without responding to them with sustained evaluation, attempts to control or suppress them, or respond to them behaviourally" (). ATT has been used with good effect for depression as a stand-alone treatment in a single case series ${ }^{15}$ as well as a component of individual ${ }^{16}$ and group MCT. ${ }^{17}$

There is promising neuropsychological and imaging evidence of the efficacy of a cognitive control training program (comprising ATT combined with the adaptive Paced Auditory Serial Attention Task) in patients with depression. ${ }^{11}$ Patients receiving cognitive control training performed significantly better than the "treatment as usual" group on executive function tests following treatment. In a later study, Siegle and colleagues reported that CCT was superior to treatment as usual in reducing levels of rumination and service utilisation in severely depressed patients. ${ }^{18}$ Due to the adjunctive nature of the cognitive 
control training program, the effects of ATT cannot be fully delineated, nevertheless those findings along with the Papageorgiou \& Wells case series, suggest that ATT may be beneficial in improving aspects of executive function in those with depression.

Given that MCT has components directly targeting executive function and attention deficits whereas CBT does not, MCT might be expected to be more effective in ameliorating these deficits. Findings in support of this would be important, as it could mean that MCT may allow for a more positive longer term prognosis. Our previous pilot study found that MCT and CBT were comparable in improving mood symptoms after 12 weeks of outpatient psychotherapy ${ }^{19}$ however post hoc analyses suggested that the disproportionate burden of social phobia comorbidity in the MCT group may have disadvantaged the MCT outcome. The current study examines the relative impact of CBT versus MCT on executive function and attentional processes in this sample. Given MCT includes the ATT, it was hypothesised that MCT would produce more rapid changes in cognitive functioning by Week 4 and would result in greater overall improvements in executive function and attention by the end of treatment, compared to CBT. 


\section{Materials and Methods}

\section{Participants}

Participants were 48 men and women aged 18-65 years with a current primary depressive episode, who were able to converse and answer questionnaires in English and provide informed consent. Exclusion criteria included bipolar I disorder, schizophrenia, current severe substance abuse, severe physical illness, an adequate course of CBT or MCT in the past year, or use of psychotropic medication (other than the occasional hypnotic). The duration of drug-free status was required to be a minimum of two weeks or five drug halflives however none in this sample were prescribed antidepressants within the previous six weeks. A research nurse screened referrals for study inclusion/exclusion criteria and potential participants were contacted by the next available therapist and booked for a clinical interview, following which informed consent was obtained from eligible participants. This study received ethical approval from the Upper South B Regional Ethics Committee, New Zealand (URB 09/03/012).

\section{Procedure}

Demographic, clinical and neuropsychological assessments were completed at pretreatment (Week 0). Clinician-rated diagnostic assessments of mood, anxiety, substance use and global assessment of functioning were conducted using the Structured Clinical Interview for the Diagnostic and Statistical Manual of Mental Disorders-IV-TR. ${ }^{20}$ Clinician ratings of current mood severity were made using the 16 item Quick Inventory of Depressive Symptomology (QIDS-C ${ }^{21}$ ) and the Montgomery Asberg Depression Rating Scale. ${ }^{22}$ Further key time points for neuropsychological assessment and ratings of depression severity were at Week 4 and at end-treatment (Week 12). 
Participants received up to 12 sessions of therapy, although a range of 8-15 sessions was permitted within the 12 weeks. Sessions were conducted twice a week for the first two weeks with the aim of six sessions within the first 4 weeks to maximise early change. Four clinical psychologists administered both forms of therapy. CBT was based on the manuals of Aaron T Beck and colleagues ${ }^{23}$ and Judith Beck. ${ }^{24}$ MCT was based on the manuals of Adrian Wells $^{12}{ }^{1}$ and Costas Papageorgiou and Wells. ${ }^{25}$

In MCT, ATT was introduced within the first two sessions and participants were asked to listen to the digital audio of ATT (available from http://www.mct-institute.com/therapistresources) twice a day for at least two weeks. ATT practice takes approximately 12 minutes and consists of three parts: focussed attention on sounds, rapid attention switching between sounds, and finally divided attention whereby the participant listens to a number of different sounds simultaneously. Participants self-rate their attentional focus pre and post treatment on a -3 (external) to +3 (internal) Likert scale. Participants were also instructed on using in vivo practice without the digital audio by using everyday sounds to practice the task.

\section{Neuropsychological assessment}

Neuropsychological assessments took approximately 90-120 minutes at weeks 0 and 12 and approximately 60 minutes at week 4 . A broad battery of neuropsychological tests was administered but apart from the National Adult Reading Test $\left(\mathrm{NART}^{26}\right)$ included as descriptive information, only tests related to our hypotheses regarding attention and executive functioning are reported here.

The Groton Maze Learning Task $\left(\mathrm{GMLT}^{27}\right)$, part of the CogState battery (www.cogstate.com), is a measure of executive function and spatial problem solving.

\footnotetext{
${ }^{1}$ Wells, A. (Unpublished). Metacognitive therapy for depression: therapist guidelines and techniques. Therapist manual. University of Manchester
} 
Participants are required (through trial and error) to find the hidden pathway between the top left corner and the lower right corner of a grid by clicking on one tile at a time but were not permitted to move diagonally, click on the same tile twice in succession or move backwards along the pathway. A tick or cross showed participants whether their move was right or wrong. The primary outcome measure was total number of errors made whilst trying to learn the pathway; with a lower score reflecting better performance.

The Controlled Oral Word Association Test $\left(\mathrm{COWAT}^{28}\right)$ is a test of verbal fluency and is used as a measure of executive function. Participants generate as many words as possible, beginning with a specific letter, over a one minute period. Different sets of letters were used at each time point.

The Rapid Visual Information Processing Task (RVIP: CANTAB ${ }^{29}$ ) is used as a measure of visual sustained attention. Participants were instructed to detect target sequences of digits (e.g., 3-5-7) from numbers 1-9 presented in a pseudo-random sequence. Primary outcome measures for the task were mean correct latency (time taken to reach a correct response) and the total number of hits.

\section{Data Analyses}

Statistical procedures were conducted using the Statistical Package for the Social Sciences (SPSS), version 22-x for Windows. ${ }^{30}$ Baseline data were analysed using independent samples t-tests and chi-squared tests. Univariate analyses of covariance (ANCOVA) were used to compare the therapy groups on change in executive function and attention measures from baseline to subsequent time points. The dependent variable in the analyses was the change in task performance from baseline to Week 4 or baseline to Week 12, therapy group was the fixed factor and baseline depression severity (QIDS) and baseline task performance, were entered as covariates. Where there was no significant difference, the 
overall change in the sample as a whole was examined. Effect sizes were calculated for the differences in change between the two therapy groups. Pearson's correlational analyses (twotailed) were used to examine the relation between change in cognitive task performance and change in depression severity.

\section{Results}

Of the 48 participants $(\mathrm{MCT} n=23, \mathrm{CBT} n=25)$ recruited for the pilot study, all completed neuropsychological testing at pre-treatment, 45 attended week 4 neuropsychological assessments (with complete data available for 43/45: MCT, $n=21$; CBT, $\mathrm{n}=22$ ); and 33 participants (MCT, $\mathrm{n}=16 ; \mathrm{CBT}, \mathrm{n}=18$ ) completed neuropsychological assessments at end treatment. Data were available then for 33/48 (69\%) of participants at all three time points. At end treatment, three participants were unable to be re-tested for logistic reasons - following the Canterbury earthquakes we were unable to access our building (and the neuropsychological testing equipment) for a number of weeks.

Demographic data reported previously in the outcome paper $^{19}$ indicated no statistically significant differences between groups for age, marital status, ethnicity or other clinical characteristics including psychiatric comorbidity which was high in this sample. Lifetime "any "anxiety prevalences were $78 \%$ in MCT and $64 \%$ in CBT groups $\left(X^{2}=1.2, d f\right.$ $=1, \mathrm{p}=0.29$ ).

\section{Insert Table 1 about here}

Data relevant to this study are reported in Table 1. At pre-treatment, key demographic or clinical characteristics did not differ between the groups. Descriptive data for the total sample were as follows: the mean years of education was 15.3 years, NART estimated pre- 
morbid IQ mean was 105 (range 83 - 118), the sample was moderately depressed, the mean age of onset was 19.8 years and number of prior depressive episodes was three.

At baseline, no statistical differences were found between the two groups with respect to any of the cognitive tasks (Table 2).

Insert Table 2 about here

\section{Neuropsychological changes from baseline to Week 4}

No statistical differences were found between the two groups for any of the neuropsychological tasks from baseline to Week 4 (Table 2). For the total sample, there was a significant reduction in total errors on the $\operatorname{GMLT}\left(\mathrm{F}_{1,41}=6.97, p=0.01\right)$ and a significant increase in the number of hits on the RVIP $\left(\mathrm{F}_{1,42}=24.11, p<0.001\right)$.

\section{Neuropsychological changes from baseline to end treatment}

There was a statistically significant difference between the two treatment modalities in extent of change on the GMLT from baseline to end treatment (Table 2). The MCT group showed a reduction in total errors but the CBT group did not (see Figure 1). Differences between the two groups for mean correct latency on the RVIP was at a trend level - latency reduced in the MCT group whereas in the CBT group, latency increased at week 12. Moderate effect sizes were seen for both the GMLT $(\mathrm{ES}=0.77, p=0.03)$ and mean correct latency on the RVIP (ES $=0.65, p=0.07$ ) although the latter was just outside significance. For the total sample, significant increases were found in the number of words generated on the COWAT $\left(\mathrm{F}_{1,32}=16.30, p<0.001\right)$ and the number of hits on the $\mathrm{RVIP}\left(\mathrm{F}_{1,32}=19.83, p\right.$ $<0.001)$ but the groups did not differ.

Insert Figure 1 about here 


\section{Relation between neuropsychological change and change in depression severity}

There were no significant correlations between change in depression severity (QIDS), and change in cognitive task performance at Week 4 (correlational coefficient range -0.179 $0.03)$ or at end treatment (correlational coefficient range $(-0.23-0.16)$. 


\section{Discussion}

The main findings of the study were as follows. By four weeks, there was little change in either group and no significant differences between the therapies in their effect on cognitive function. At end treatment however, there was a significantly greater improvement in performance on the GMLT (spatial working memory and executive function) in the MCT group compared with the CBT group. The effect size of the difference was 0.77 . There was also a moderate effect size difference in favour of MCT on an attentional task (RVPT latency); however, this was only at a trend level. Changes in executive function and attention did not correlate with changes in mood. The outcome of our previously published pilot study was that both therapies were effective but did not differ in improving depression severity ${ }^{19}$, although the greater comorbidity burden in MCT is likely to have affected those results. Considered together, these findings add to previous research suggesting only a limited relationship between mood change and neuropsychological change during treatment of depression. $^{31}$

As noted earlier, executive functioning comprises a variety of different neuropsychological processes. Pietrzak and colleagues ${ }^{32}$ suggested that the total errors score on the GMLT is likely to be a measure of psychomotor speed, working memory and attention. Specific targeting of attentional processes in MCT might account for the relatively greater improvement on both the GMLT and trend level finding for RVIP tasks in the MCT group through improved attentional control, relative to other executive function tests such as the COWAT which assess other domains of executive function.

There is a growing literature examining changes in brain function after therapies such as CBT for a range of emotional disorders, with increasing numbers of imaging studies detecting improvement after psychotherapy (e.g. ${ }^{33-35}$ ). Relatively few previous studies have 
examined the effects of psychotherapy on neuropsychological functioning in depression. One large study $(n=272)$ of young adults by Bastos and colleagues ${ }^{36}$ reported greater improvement on measures of working memory, processing speed and executive function after 24 months of psychodynamic therapy compared to fluoxetine or a combination of the two treatments. This suggests that for those without chronic illness, psychotherapy (even without specific cognitive remediation strategies) may improve cognition and to a greater extent than medication.

There is increased interest in the use of cognitive remediation strategies, some of which resemble ATT, although many batteries are broader in scope (see Porter et $\mathrm{al}^{37}$ review). These studies suggest a positive effect of these interventions on neuropsychological function in patients with lifetime or current depression. As noted earlier though, domains such as executive function comprise a number of related but distinguishable domains and the changes found tend to be focal changes and related to the specific training interventions rather than global neuropsychological benefits. ${ }^{1,38}$

These preliminary findings suggest MCT may have an advantage over CBT in improving certain aspects of executive function, particularly attention. This finding could be seen as being consistent with that of Siegle and colleagues of a specific benefit of a cognitive control strategy (including ATT as a component) in improving executive function processes, compared to their active "treatment as usual" group. ${ }^{11}$ A recent study found that ATT applied to traumatic stress symptoms in a non-clinical population resulted in improved self-reported attentional flexibility and improved attentional performance on a neuropsychological test of emotional set shifting task compared to a control intervention group. ${ }^{39}$ Together these findings could be seen to provide preliminary support for the purported mechanism of action in MCT; that is, that attentional training and improving control over thinking is associated with beneficial effects on executive functioning and attention which enhances the capacity to 
disengage from the cognitive attentional syndrome. ${ }^{12,39}$ This supports other literature suggesting a specific action of ATT in increasing attentional flexibility enabling attentional disengagement, with corresponding effects on subcortical correlates of emotional regulation strategies. $^{40}$

As noted earlier, persistent cognitive deficits have been found in those with a history of mood disorders even when acute mood symptoms are improved to subthreshold levels. ${ }^{4}$ This combined with the present finding of the independence of mood and neuropsychological change suggests that the clinical benefits of cognitive remediation may be in domains other than mood. Improved executive functioning and attention control is likely to have positive impacts in improved life functioning such as academic or occupational functioning but may also benefit interpersonal functioning through reducing rumination and excessive selffocussed attention.

There are several limitations to the current study. This between-therapy examination of neuropsychological change is a secondary analysis of data from our earlier therapy comparison pilot study whose outcome was improved depression severity and which was not adequately powered to detect small or moderate effect sizes. The sample size issue was exacerbated by the earthquake interruption preventing the last three end assessments being conducted. Given previous findings from a larger sample of depressed patients who differed from control subjects in performance on similar tasks of 0.5 to $0.7^{41}$, this pilot study was unlikely to give anything more than an estimate of between therapy effect size differences to inform the design of a larger study. Despite this, differences were found between MCT and CBT. The effect sizes of $0.65-0.77$ in this study for the RVIP latency and GMLT errors respectively suggest that a future study examining neuropsychological functioning should have between 50 and 60 participants per group. 
Given the exploratory nature of this study, correction was not made for multiple comparisons; hence, it is possible that the difference on the GMLT is a chance finding. It would have been preferable to use repeated measures analysis of variance design with time as a factor. However, the decision to analyse change to Week 4 and to Week 12 separately took into account missing data at each time point due to attrition between 4 and 12 weeks. The possibility of selective drop-out of subjects with more or less neuropsychological change has the potential to affect the findings on overall change; however, we consider that this is unlikely to differ between groups given comparable pre-treatment data and dropout rates. Although we were interested in early change, it may have been optimistic to expect to detect neuropsychological change by 4 weeks, although this clearly occurs by 12 weeks. Neuropsychological functioning was not re-assessed at follow-up so we are unable to assess how long those changes persist after the end of treatment.

This pilot study has a number of strengths. To our knowledge, this is the first study to examine the effects of MCT and CBT on neuropsychological functioning in depressed individuals. In addition these findings add to the limited literature on neuropsychological change associated with psychotherapy for depression. Participants were free of psychotropic medication so medication effects can be ruled out as potential confounders. Furthermore the majority of participants were referred to the study via health professionals and therefore are likely to reflect individuals found in a true clinical sample. The study provides effect size data to assist in powering larger, more definitive studies. 


\section{Conclusions}

This exploratory study suggests that independent of changes in depressive severity, MCT (which includes ATT) may have an advantage over CBT in improving neuropsychological functioning where a high attentional capacity is required. The comparable between-therapy improvements for other executive function tasks is not unexpected given previous research indicating that specific interventions (such as ATT) tend to improve related (focal) executive functions rather than global improvement across other domains. However given the central role of attention in underpinning other cognitive functioning, targeting attention is clearly a worthwhile objective. These preliminary findings of specific benefit of MCT in aspects of neuropsychological functioning (along with the earlier suggestion that MCT may have been disadvantaged by comorbidity burden) are indications of the urgent need for further research using larger samples stratifying for potential confounders. Future research should address longer term neuropsychological outcomes as well as how changes on tests of neuropsychological functioning translate to real world mood and functional outcomes. 


\section{Acknowledgements}

We thank Julia Martin, Robyn Abbott, Andrea Bartram, Bridget Kimber, Steff Watts and Megan Tucker for their invaluable assistance with the research, the University of Otago, Christchurch and Canterbury District Health Board for their support, and funders the New Zealand Lottery Board Health fund (grant 279032); and the University of Otago Research Fund (grant ORG0109-0310). Funders had no input into the design, undertaking of the study or the writing of this paper. 


\section{References}

1. Miyake A, Friedman NP, Emerson MJ, et al. The unity and diversity of executive functions and their contributions to complex "frontal lobe" tasks: a latent variable analysis. Cognitive Psychol. 2000;41(1):49-100.

2. Porter RJ, Bourke C, Gallagher P. Neuropsychological impairment in major depression: its nature, origin and clinical significance. Aust NZ J Psychiat. 2007;41(2):115-28.

3. Douglas KM, Porter RJ, Knight RG, et al. Neuropsychological changes and treatment response in severe depression. Brit J Psychiat. 2011;198:115-22.

4. Reppermund S, Zihl J, Lucae S, et al. Persistent cognitive impairment in depression: The role of psychopathology and altered hypothalamic-pituitary-adrenocortical (HPA) system regulation. Bol Psychiat. 2007;62:400-6.

5. McIntyre RS. Cognitive dysfunction in major depressive disorders: affected domains and impact on functioning. CNS \& Neurol Dis- Drug Targets. In Press.

6. Withall A, Harris LM, Cumming SR. The relationship between cognitive function and clinical and functional outcomes in major depressive disorder. Psychol Med. 2009;39(3):393-402.

7. Alexopoulos GS, Meyers BS, Young RC, et al. Executive dysfunction and long-term outcomes of geriatric depression. Arch Gen Psychiat. 2000;57(3):285-90.

8. Priemer M, Talbot F. CBT guided self-help compares favourably to gold standard therapistadministered CBT and shows unique benefits over traditional treatment. Behav Change. 2013;30:227-40.

9. Butler AC, Chapman JE, Formanc EM, et al. The empirical status of cognitive-behavioral therapy: A review of meta-analyses Clin Psychol Rev. 2006;26(1):17-31.

10. Beck AT. The current state of cognitive therapy: a 40-year retrospective. Arch Gen Psychiat. 2005;62:953-9.

11. Siegle GJ, Ghinassi F, Thase ME. Neurobehavioral therapies in the 21st century: summary of an emerging field and an extended example of cognitive control training for depression. Cognitive Ther Res. 2007;31(2):235-62.

12. Wells A. Metacognitive therapy for anxiety and depression. New York: Guilford Press; 2009. 13. Wells A. Panic disorder in association with relaxation-induced anxiety: an attention training approach to treatment. Behav Ther. 1990;21:273-80.

14. Wells A. Detached mindfulness in cognitive therapy: A metacognitive analysis and ten techniques. Journal of Rational-Emotive \& Cognitive Behavior Therapy. 2005;23(4):337-55.

15. Papageorgiou C, Wells A. Treatment of recurrent major depression with attention training. Cogn Behav Pract. 2000;7:407-13.

16. Wells $A$, Fisher $P$, Myers $S$, et al. Metacognitive therapy in treatment-resistant depression: $A$ platform trial. Behav Res Ther. 2012;50:367-73.

17. Dammen T, Papageorgiou C, Wells A. An open trial of group metacognitive therapy for depression in Norway. Nord J Psychiat. 2014(0):1-6.

18. Siegle GJ, Price RB, Jones NP, et al. You Gotta Work at It Pupillary Indices of Task Focus Are Prognostic for Response to a Neurocognitive Intervention for Rumination in Depression. Clin Psychol Sci. 2014;2(4):455-71.

19. Jordan J, Carter JD, McIntosh VV, et al. Metacognitive therapy versus cognitive behavioural therapy for depression: a randomized pilot study. Aust NZ J Psychiat. 2014;OnlineFirst May 82014.

20. First MB, Spitzer RL, Gibbon M, et al. Structured Clinical Interview for DSM-IV-TR Axis I Disorders-Patient Edition (SCID-I/P. 2/2001 Revision). New York: Biometrics Research Department, New York State Psychiatric Institute. 2001.

21. Rush AJ, Trivedi MH, Ibrahim HM, et al. The 16-Item Quick Inventory of Depressive Symptomatology (QIDS), clinician rating (QIDS-C), and self-report (QIDS-SR): a psychometric evaluation in patients with chronic major depression. Biol Psychiatry. 2003;54(5):573-83. 
22. Montgomery SA, Asberg M. A new depression scale designed to be sensitive to change. Brit J Psychiat. 1979;134:382-9.

23. Beck AT, Rush AJ, Shaw BF, et al. Cognitive therapy of depression: A treatment manual. Philadelphia: Center for Cognitive Therapy; 1978.

24. Beck JS. Cognitive therapy: basics and beyond. New York: The Guilford Press; 1995.

25. Papageorgiou C, Wells A, editors. Depressive rumination: nature, theory and treatment. West Sussex, England: John Wiley; 2004.

26. Nelson HE. National Adult Reading Test (NART): For the assessment of premorbid intelligence in patients with dementia: Test manual: NFER-Nelson; 1982.

27. Snyder PJ, Jackson CE, Piskulic D, et al. Spatial working memory and problem solving in schizophrenia: the effect of symptom stabilization with atypical antipsychotic medication. Psychiatry Research. 2008;160(3):316-26.

28. Benton AL, Hamsher K. Multilingual Aphasia Examination. lowa City: University of lowa; 1976.

29. Cambridge Cognition Limited. CANTABeclipse (Available from: http://www.cantabeclipse.com). 2007.

30. IBM Corp. SPSS Statistics for Windows. 19.0 ed. Armonk, NY: IBM Corp; 2010.

31. Douglas KM, Porter RJ. Longitudinal assessment of neuropsychological function in major depression. Aust NZ J Psychiat. 2009;43(12):1105-17.

32. Pietrzak RH, Maruff $P$, Mayes LC, et al. An examination of the construct validity and factor structure of the Groton Maze Learning Test, a new measure of spatial working memory, learning efficiency, and error monitoring. Arch Clin Neuropsych. 2008;23(4):433-45.

33. Paquette V, Lévesque J, Mensour B, et al. "Change the mind and you change the brain": effects of cognitive-behavioral therapy on the neural correlates of spider phobia. Neuroimage. 2003;18(2):401-9.

34. Linden D. How psychotherapy changes the brain-the contribution of functional neuroimaging. Mol Psychiatr. 2006;11(6):528-38.

35. Goldapple K, Segal Z, Garson C, et al. Modulation of cortical-limbic pathways in major depression: treatment-specific effects of cognitive behavior therapy. Arch Gen Psychiat. 2004;61(1):34-41.

36. Bastos AG, Guimaraes LS, Trentini CM. Neurocognitive changes in depressed patients in psychodynamic psychotherapy, therapy with fluoxetine and combination therapy. J Affect Disorders. 2013;151(3):1066-75.

37. Porter RJ, Douglas K, Jordan J, et al. Psychological treatment for cognitive dysfunction in major depressive disorder; current evidence and perspectives. CNS \& Neurol Dis- Drug Targets. In Press.

38. Mackin RS, Nelson JC, Delucchi K, et al. Cognitive outcomes after psychotherapeutic interventions for major depression in older adults with executive dysfunction. Am J Geriat Psychiat. 2013.

39. Callinan S, Johnson D, Wells A. A randomised controlled study of the effects of the attention training technique on traumatic stress symptoms, emotional attention set shifting and flexibility. Cognit Ther and Res. 2014;Online First 6 August 2014.

40. Siegle GJ, Ghinassi F, Thase ME. Neurobehavioral therapies in the 21st century: summary of an emerging field and an extended example of Cognitive Control Training for depression. Cognit Ther Res. 2007; Volume 31(2):235-62.

41. Bourke C, Porter RJ, Carter JD, et al. Comparison of neuropsychological functioning and emotional processing in major depression and social anxiety disorder subjects, and matched healthy controls. Aust NZ J Psychiat. 2012;46(10):972-81. 
Table 1. Pre-treatment functioning and psychiatric comorbidity in metacognitive therapy (MCT) and cognitive behavior therapy (CBT) groups.

\begin{tabular}{lccccccc}
\hline & $\begin{array}{c}\text { MCT } \\
\text { Mean } \\
\text { or \% }\end{array}$ & SD & $\begin{array}{c}\text { CBT } \\
\text { Mean } \\
\text { or \% }\end{array}$ & SD & Statistic $^{\text {a }}$ & $\boldsymbol{p}$ \\
& $\mathbf{n = 2 3}$ & & $\boldsymbol{n = 2 5}$ & & & \\
& & & & & & \\
\hline Demographics & 37.2 & 12.7 & 35.0 & 13.0 & 0.60 & 0.55 \\
Age (years) & $52: 48$ & & $52: 48$ & & & \\
Gender (Male: Female) & 14.6 & 2.7 & 14.5 & 2.6 & 1.67 & 0.87 \\
Education (Mean years) & 105.5 & 6.6 & 104.5 & 8.3 & 0.44 & 0.66 \\
NART IQ & & & & & &
\end{tabular}

\section{Clinical}

QIDS

$\begin{array}{llllll}15.3 & 4.1 & 15.0 & 4.5 & 0.24 & 0.81\end{array}$

MADRS

24.7

7.7

22.6

7.2

$0.96 \quad 0.34$

Age onset of depression

$22.5 \quad 16.2$

17.4

19.1

1.32

0.19

Prior depressive episodes

4.2

3.7

3.4

2.7

0.80

0.43

Note: $\quad{ }^{a}$ T-tests for continuous variables or chi-square for dichotomous variables.

Abbreviations: Standard deviation (SD); National Adult Reading Test intelligence quotient (NART IQ); Quick inventory of depressive symptomatology- clinician-rated (QIDS); Montgomery Asberg Depression Rating Scale (MADRS). 
Table 2. Neuropsychological performance at baseline, 4 weeks and end treatment (12 weeks) in metacognitive therapy (MCT) and cognitive behavior therapy (CBT) groups.

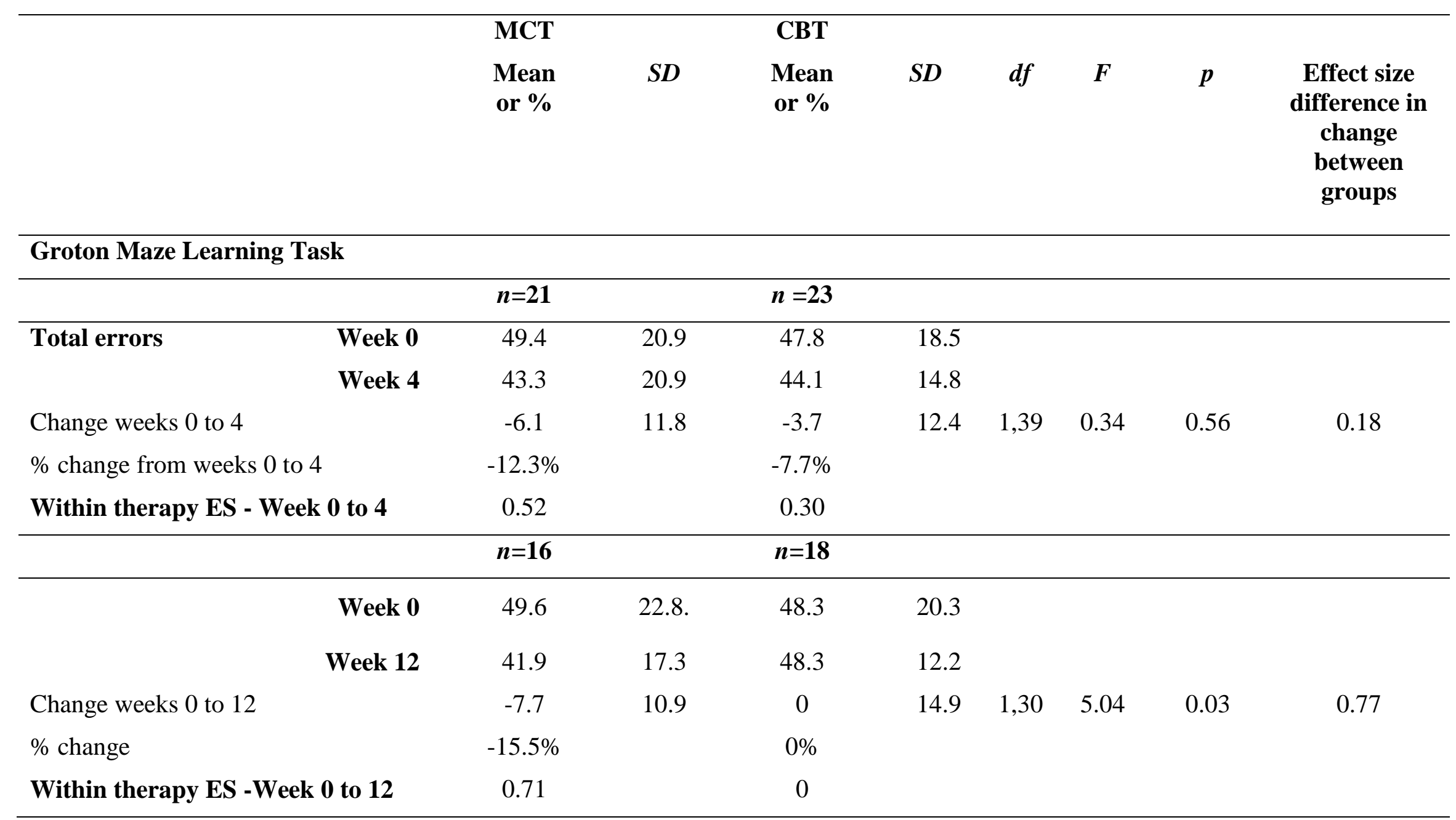


Table 2 (cont.). Neuropsychological performance at baseline, 4 weeks and end treatment (12 weeks) in metacognitive therapy (MCT) and cognitive behavior therapy (CBT) groups.

\begin{tabular}{|c|c|c|c|c|c|c|c|c|}
\hline & MCT & & CBT & & & & & \\
\hline & $\begin{array}{c}\text { Mean } \\
\text { or \% }\end{array}$ & $S D$ & $\begin{array}{l}\text { Mean } \\
\text { or \% }\end{array}$ & $S D$ & $d f$ & $\boldsymbol{F}$ & $p$ & $\begin{array}{l}\text { Effect size } \\
\text { difference in } \\
\text { change } \\
\text { between } \\
\text { groups }\end{array}$ \\
\hline \multicolumn{9}{|l|}{ Controlled Oral Word Association Task } \\
\hline & $n=22$ & & $n=23$ & & & & & \\
\hline \multirow[t]{2}{*}{ Sum of words } & 42.6 & 8.7 & 41.0 & 13.2 & & & & \\
\hline & 42.1 & 10.5 & 41.4 & 10.6 & & & & \\
\hline Change weeks 0 to 4 & -0.5 & 5.50 & 0.4 & 6.2 & 1,41 & 0.16 & 0.68 & 0.12 \\
\hline$\%$ change from weeks 0 to 4 & $-1.2 \%$ & & $1.0 \% \%$ & & & & & \\
\hline \multirow[t]{2}{*}{ Within therapy ES - Week 0 to 4} & 0.10 & & 0.06 & & & & & \\
\hline & $n=16$ & & $n=18$ & & & & & \\
\hline Week 0 & 41.1 & 9.4 & 41.3 & 14.2 & & & & \\
\hline Week 12 & 43.9 & 9.9 & 47.2 & 14.6 & & & & \\
\hline Change weeks 0 to 12 & 2.6 & 7.3 & 5.9 & 5.3 & 1,30 & 2.06 & 0.16 & 0.49 \\
\hline$\%$ change & $6.8 \%$ & & $14.3 \%$ & & & & & \\
\hline Within therapy ES -Week 0 to 12 & 0.38 & & 1.12 & & & & & \\
\hline
\end{tabular}


Table 2 (cont.). Neuropsychological performance at baseline, 4 weeks and end treatment (12 weeks) in metacognitive therapy (MCT) and cognitive behavior therapy (CBT) groups.

\begin{tabular}{|c|c|c|c|c|c|c|c|c|}
\hline & MCT & & CBT & & & & & \\
\hline & $\begin{array}{l}\text { Mean } \\
\text { or \% }\end{array}$ & $S D$ & $\begin{array}{c}\text { Mean } \\
\text { or \% }\end{array}$ & $S D$ & $d f$ & $\boldsymbol{F}$ & $p$ & $\begin{array}{c}\text { Effect size } \\
\text { difference in } \\
\text { change } \\
\text { between } \\
\text { groups }\end{array}$ \\
\hline \multicolumn{9}{|l|}{ Rapid Visual Information Processing } \\
\hline & $n=22$ & & $n=22$ & & & & & \\
\hline \multirow[t]{2}{*}{ Hits } & 18.5 & 4.7 & 18.4 & 5.2 & & & & \\
\hline & 21.0 & 3.7 & 20.9 & 4.6 & & & & \\
\hline Change weeks 0 to 4 & 2.5 & 3.8 & 2.5 & 3.0 & 1,40 & $<0.01$ & 0.96 & 0.01 \\
\hline$\%$ change from weeks 0 to 4 & $13.5 \%$ & & $13.6 \%$ & & & & & \\
\hline \multirow[t]{2}{*}{ Within therapy ES - Week 0 to 4} & 0.67 & & 0.84 & & & & & \\
\hline & $n=16$ & & $n=18$ & & & & & \\
\hline Week 0 & 18.2 & 4.58 & 17.4 & 5.5 & & & & \\
\hline Week 12 & 21.9 & 4.56 & 19.9 & 6.4 & & & & \\
\hline Change weeks 0 to 12 & 3.7 & 3.96 & 2.5 & 4.1 & 1,30 & 1.07 & 0.31 & 0.35 \\
\hline$\%$ change & $20.3 \%$ & & $14.4 \%$ & & & & & \\
\hline Within therapy ES -Week 0 to 12 & 0.93 & & 0.60 & & & & & \\
\hline
\end{tabular}


Table 2 (cont.). Neuropsychological performance at baseline, 4 weeks and end treatment (12 weeks) in metacognitive therapy (MCT) and cognitive behavior therapy (CBT) groups.

\begin{tabular}{|c|c|c|c|c|c|c|c|c|c|}
\hline & & \multicolumn{2}{|l|}{ MCT } & \multicolumn{2}{|l|}{ CBT } & \multirow[b]{2}{*}{$d f$} & \multirow[b]{2}{*}{$\boldsymbol{F}$} & \multirow[b]{2}{*}{$p$} & \multirow[b]{2}{*}{$\begin{array}{c}\text { Effect size } \\
\text { difference in } \\
\text { change } \\
\text { between } \\
\text { groups }\end{array}$} \\
\hline & & $\begin{array}{c}\text { Mean } \\
\text { or } \%\end{array}$ & $S D$ & $\begin{array}{l}\text { Mean } \\
\text { or } \%\end{array}$ & $S D$ & & & & \\
\hline \multicolumn{10}{|c|}{ Rapid Visual Information Processing } \\
\hline & & $n=22$ & & $n=\mathbf{2 3}$ & & & & & \\
\hline \multirow[t]{2}{*}{ Mean Correct Latency } & Week 0 & 411.9 & 76.9 & 407.9 & 76.9 & & & & \\
\hline & Week 4 & 400.6 & 63.8 & 400.2 & 81.4 & & & & \\
\hline Change weeks 0 to 4 & & -11.3 & 68.9 & -7.7 & 69.8 & 1,40 & $<0.01$ & 0.97 & 0.01 \\
\hline$\%$ change from weeks 0 & & $-2.7 \%$ & & $-1.9 \%$ & & & & & \\
\hline \multirow{2}{*}{\multicolumn{2}{|c|}{ Within therapy ES - Week 0 to 4}} & 0.16 & & 0.11 & & & & & \\
\hline & & $n=16$ & & $n=18$ & & & & & \\
\hline & Week 0 & 417.6 & 77.5 & 405.5 & 78.7 & & & & \\
\hline & Week 12 & 381.1 & 52.4 & 423.6 & 119.9 & & & & \\
\hline Change weeks 0 to 12 & & -36.5 & 76.2 & 18.1 & 81.5 & 1,30 & 3.60 & 0.07 & 0.65 \\
\hline$\%$ change & & $-8.7 \%$ & & $4.5 \%$ & & & & & \\
\hline Within therapy ES -We & 0 to 12 & 0.48 & & 0.22 & & & & & \\
\hline
\end{tabular}

Notes: Abbreviations: SD: standard deviation. ES: effect size (Cohen's $d$ ) 
Figure 1 Changes on the Groton Maze Learning Test following metacognitive therapy (MCT) or cognitive behaviour therapy (CBT) for depression 